\title{
Neurite Growth on Different Substrates: Permissive versus Instructive Influences and the Role of Adhesive Strength
}

\author{
Vance Lemmon, ${ }^{1}$ Susan M. Burden, ${ }^{1}$ H. Ross Payne, ${ }^{1}$ Gwendolynne J. Elmslie, ${ }^{1}$ and Mary Louise Hlavin ${ }^{1,2}$ \\ 'Department of Neurosciences and 'Department of Neurosurgery, Case Western Reserve University, Cleveland, \\ Ohio 44106-4975
}

\begin{abstract}
Growing axons use environmental cues to guide them to their targets. One class of cues is thought to be adhesion molecules on cells and in the extracellular matrix that axons interact with as they grow to their targets. In choosing between two possible pathways, the relative adhesiveness of the two substrates could be an important factor in controlling neurite growth. We conducted experiments in vitro to study how naturally occurring adhesion molecules influence neurite growth. Neurite growth rates, the degree of neurite fasciculation, the choices neurites make between two substrates, and the relative adhesiveness of different substrates were examined. We found that the relative adhesiveness of a substrate was a poor predictor of either axon growth rate or the degree of fasciculation. Furthermore, neurites showed little selectivity between three different naturally occurring substrates, L1, $\mathrm{N}$-cadherin, and laminin. These results suggest that some adhesion molecules may serve as permissive substrates in that they can define axonal pathways but they do not provide information about which path to take at a choice point or about which direction to go along the path. Finally, these results suggest that substrates in vivo may not exert their effects on axon guidance principally via relative adhesiveness.
\end{abstract}

Understanding how axons are guided to their targets is a central problem in developmental neurobiology. Several different mechanisms have been proposed to subserve this function. These include diffusible tropic molecules, channels, or substrate molecules patterned to provide pathways to targets. Substrate-bound adhesion molecules, such as cell adhesion molecules (CAMs) or extracellular matrix moleculcs, could rcgulatc axon growth through a variety of mechanisms. Letourneau (1975) performed an important experiment that indicated axons might choose pathways based on simple adhesive hierarchies. He found that axons prefer to grow on polyornithine and collagen rather than palladium-coated surfaces and that axons prefer palladium to tissue culture plastic; these preferences correlated with the strength of binding of the growth cones to the various surfaces. The positive correlation between adhesivity and growth cone selectivity established by these studies has been widely inter-

\footnotetext{
Received July 24, 1991; revised Oct. 14, 1991; accepted Oct. 18, 1991.

We thank F. Bonhoeffer for providing the silicon matrix used to construct the parallel lanes in the choice experiments and $M$. Takeichi and $G$. Grunwald for providing the NCD-2 hybridoma cell line. We also thank the reviewers for their thoughtful comments. This work was supported by National Eye Institute Grant 5285 .

Correspondence should be addressed to Vance Lemmon at the above address. Copyright @ 1992 Society for Neuroscience 0270-6474/92/120818-09\$05.00/0
}

preted to indicate that axons in vivo choose between different paths by growing along the more adhesive one (for reviews, see Lockerbie, 1987; Sanes, 1989).

Over the past few years, several observations have been made that suggest that substrate molecules can have complex influences on neurite growth. For example, leech neurons in vitro show no selectivity for growth on two different substrates but the morphology of the neurites (Chiquet and Nicholls, 1987) and the localization of $\mathrm{Ca}^{2+}$ channels on the neurites (Ross et al., 1988) differ depending on the substrate. Similarly, neurites of sympathetic neurons in vitro express either axonal or dendritic properties depending on the substrate (Lein and Higgins, 1989). These observations indicate that a substrate can have an instructive influence on neurite growth, causing dramatic alterations in plasma membrane and cytoskeletal composition. Neurites have been found not to show oriented growth when extending over a gradient of laminin (McKenna and Raper, 1988), and the rate of growth and neurite initiation is relatively insensitive to laminin concentration (Buettner and Pittman, 1991). Such findings suggest that some substrate molecules may be permissive, inducing neurite elongation, but not instructive, in the sense of directing growth in a particular direction along a pathway or indicating which way to go at an intersection between two pathways. Substrates also may have an inhibitory influence on neurite outgrowth. Bonhoeffer and associates have shown that temporal retinal axons, when given a choice between anterior and posterior tectal membranes, prefer to grow on membranes from anterior tectum, and that this selectivity is due to the presence of an inhibitory substance on the posterior tectum (Walter et al., 1987). Similarly, proteoglycans (Snow et al., 1990) and proteins produced by oligodendrocytes (Schwab and Schnell, 1991) have been shown to inhibit axon growth in ways that can direct axon pathway selection. Finally, dorsal root ganglion neurites prefer laminin to collagen yet their growth cones bind more weakly to laminin than collagen (Gundersen, 1987), suggesting that a neurite's response to a substrate is independent of its adhesiveness.

In the studies reported here, we have examined three of the CAMs and extracellular matrix molecules that promote neurite growth. We compared the ability of $\mathrm{L1}, \mathrm{N}$-cadherin, and laminin, as well as poly-L-lysine (PL), to promote neurite outgrowth, to affect the degree of neurite fasciculation, and to influence the choices neurites make between substrates with different adhesivities for growth cones. L1 and N-cadherin were chosen because of their presence on axons, their proven ability to support neurite growth, and their likely importance in axon growth and fascicle formation in vivo. Laminin was tested because of its potent neurite growth-promoting properties and its presence in 
developing axon pathways. We tested it in two presentations, either on nitrocellulose or PL because (1) nitrocellulose was used to adhere the two CAMs yet many other investigators use PL to increase laminin binding to plastic and (2) preliminary experiments showed that neurites responded slightly differently to laminin with the two different methods. PL was used because it represents the interesting case of a substrate that supports neurite growth yet probably does this despite the absence of a bona fide cell surface receptor on neurites for this substrate. Moreover, PL is widely used as a substrate for neuronal attachment in cell culture experiments. If growth cone behavior is determined principally by degree of adhesion to a given substrate, then PL should be a good substrate for predicting growth cone behavior. To interpret the following experiments, it was crucial that the experiments be done on a relatively homogeneous population of growth cones. For this reason, the very long neurites that emerge from chick retinal explants were chosen. They grow from retinal ganglion cells, and the neurites express axonal markers such as $\mathrm{L} 1, \mathrm{~F} 11$, neurofascin, and phosphorylated neurofilament.

We found that the adhesivity of a substrate was not correlated with the degree of neurite fasciculation or growth rates. A major and unanticipated finding was that neurites showed little selectivity when permitted to choose between $\mathrm{L} 1, \mathrm{~N}$-cadherin, or laminin. Because $\mathrm{Ll}$ is much more adhesive than $\mathrm{N}$-cadherin or laminin, this suggests that relative substrate adhesiveness is not a principle factor in guiding axon growth.

\section{Materials and Methods}

Preparation of substrates. Nitrocellulose was obtained from Schleicher and Schuell. Laminin was obtained from GIBCO, Collaborative Research, or Upstate Biotechnologies. Poly-L-lysine (PL) was obtained from Sigma. Rat $\mathrm{L} 1$ and chick $\mathrm{L} 1$ were purified using an affinity column conjugated to 74-5H7 (Lemmon et al., 1989) or 8D9 (Lemmon and McLoon, 1986) antibodies, respectively. $\mathrm{N}$-cadherin was purified using antibody NCD-2 (Hatta and Takeichi, 1986) as described by Bixby and Zhang (1990).

Corning $35 \mathrm{~mm}$ tissue culture dishes were coated with a solution of nitrocellulose (NC) dissolved in methanol (Lagenaur and Lemmon, 1987). Laminin dishes were coated with a $1 \mathrm{mg} / \mathrm{ml}$ solution of laminin in $\mathrm{Ca}^{2+}$. $\mathrm{Mg}^{2+}$-free Hank's buffer (CMF). Rat $\mathrm{Ll}$, chick $\mathrm{L} 1$, and $\mathrm{N}$-cadherin were used, with protein concentrations of $0.8,0.3$, and $0.1 \mathrm{mg} / \mathrm{ml}$, respectively, determined by the Pierce BCA protein assay. These concentrations of substrates were assumed to produce a saturating effect on growth cone behavior becausc 10-fold dilutions of the substrates resulted in identical responses from the neurites. Substrates were applied by spreading $20 \mu \mathrm{l}$ of substrate solution across an NC-coated dish, followed by blocking with $1 \mathrm{ml}$ of fetal bovine serum (FBS) and storage in $1.5 \mathrm{ml}$ of plating medium. Plating medium consisted of Dulbecco's modified Eagle's medium (DMEM) $/ 10 \%$ FBS $/ 5 \%$ chick embryo extract (CEE) and penicillin/streptomycin/fungizone (PSF). Dishes were incubated at $37^{\circ} \mathrm{C}$ in $5 \% \mathrm{CO}_{2}, 95 \%$ air while retinal explants were prepared.

Poly-L-lysine dishes were prepared by incubating $35 \mathrm{~mm}$ tissue culture dishes in $0.1 \mathrm{mg} / \mathrm{ml}$ PL in sterile distilled water for $3-4 \mathrm{hr}$ at $37^{\circ} \mathrm{C}$ in $5 \% \mathrm{CO}_{2}, 95 \%$ air. These dishes were rinsed twice with $\mathrm{CMF}$ and incubated in plating medium until used for retinal explant plating. For laminin-PL dishes, PL-coated dishes were incubated with a solution of $10 \mu \mathrm{g} / \mathrm{ml}$ laminin in CMF at $37^{\circ} \mathrm{C}$ in $5 \% \mathrm{CO}_{2}, 95 \%$ air. The laminin solution was then replaced with plating medium and incubated during preparation of retinal explants.

Preparation of retinal explant strips. Fertilized White Leghorn chicken eggs were incubated in a humidified, forced-air incubator at $39^{\circ} \mathrm{C}$. Embryonic day 7 or 8 (E7 or E8, corresponding to Hamburger and Hamilton stages 28-29) embryos were used for cultures. The procedure for retinal explant cultures has been previously described (Halfter et al., 1983; Drazba and Lemmon, 1990). Retinal explant strips were incubated at $37^{\circ} \mathrm{C}$ in $5 \% \mathrm{CO}_{2}, 95 \%$ air for $40-48 \mathrm{hr}$ before use for growth rates or blasting experiments.
Growth rate assessment. Neurite growth rates on the various substrates were assayed with time-lapse video microscopy. Cultures were removed from the incubator, and either additional prewarmed $\left(37^{\circ} \mathrm{C}\right)$ plating medium (DMEM/10\% FBS/5\% CEE/PSF) was added or the medium was exchanged with prewarmed HEPES-DMEM $/ 10 \% \mathrm{FBS} / 5 \% \mathrm{CEE} /$ PSF ( $\mathrm{pH} 7.1$ ). Dishes were then coverslipped and placed in a heated stage ring of a Sensortek TS-4 temperature controller on a Leitz Diavert microscope. The ring maintained the culture medium temperature at $37^{\circ} \mathrm{C}$. The microscope was in a Plexiglas incubation chamber to decrease temperature variability. A continuous flow of $5 \% \mathrm{CO}_{2}, 95 \%$ air was pumped into the incubation chamber if the dish contained normal plating medium. Time-lapse video images were obtained with a Javelin Chromachip II camera and Javelin Heliquad time-lapse video recorder at setting 216 . Under these conditions, cultures remained healthy for greater than $8 \mathrm{hr}$ of recording time, although most recording sessions were limited to $8 \mathrm{hr}$ maximum per dish. Neurite growth was reviewed on an NEC monitor, transparency traces were made at approximately 30 min intervals, and the distance was measured between time points with a Bioquant Image Analysis System IV. A 10× objective was used for all measurements, and growth rates were assessed as micrometers of growth per hour. Growth cones used for growth rate analysis were limited to single growth cones that grew unobstructed and without retraction during the videotaping.

Fasciculation assessment by scanning electron microscopy. Explants with neurites were rinsed briefly in fresh medium cooled to $4^{\circ} \mathrm{C}$. The explants were then fixed for $2 \mathrm{hr}$ at $4^{\circ} \mathrm{C}$ in $4 \%$ glutaraldehyde $/ 4 \%$ sucrose in $0.1 \mathrm{~m}$ sodium cacodylate buffer, $\mathrm{pH} 7.4$. The fixed specimens were washed for $1 \mathrm{hr}$ at $24^{\circ} \mathrm{C}$ with $4 \%$ sucrose in $0.1 \mathrm{~m}$ sodium cacodylate,

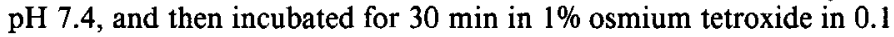
$M$ cacodylate buffer. A conductive coating was chemically deposited onto the specimen surface by two cycles of $15 \mathrm{~min}$ incubations in (1) $1 \%$ tannic acid buffered with $0.1 \mathrm{~m}$ sodium cacodylate, $\mathrm{pH} 7.4$, followed by (2) $1 \%$ osmium tetroxide in $0.1 \mathrm{M}$ sodium cacodylate (Postek et al., 1980). The specimens were dehydrated in an ascending ethanol series, transferred from $100 \%$ ethanol to Freon 113 , and dried in a critical point drying apparatus. The dried coverslips were mounted onto aluminum stubs, coated with gold, and viewed with a scanning electron microscope. Neurite fasciculation on each substrate was assessed visually, and no obvious change in fasciculation was detected between 24 and $48 \mathrm{hr}$ cultures on the same substrate. A quantitative assessment of fasciculation was made by photographing five random fields on each substrate at $800 \times$. The widths of fascicles in each field were measured.

Growth cone blasting/adhesivity assay. Adhesivity of retinal ganglion cell growth cones on various substrates was measured using a modification of an assay previously described (Gundersen and Barrett, 1980). Explants in culture $40-48 \mathrm{hr}$ were used to correspond to the growth rate data. Dishes were removed from incubation, the medium was exchanged with prewarmed $\left(37^{\circ} \mathrm{C}\right.$ ) HEPES-DMEM $/ 10 \% \mathrm{FBS} / 5 \%$ CEE/PSF (pH 7.1 ), and the dishes were placed in the same microscope system used to measure growth rates. Individual growth cones were observed using a $20 \times$ objective, and a grid eyepiece was used to measure the distance from the lcading edge of the growth concs and the pipctte tip.

The blasting apparatus consisted of a 1-mm-diameter capillary pipette pulled to a $1.5 \mu \mathrm{m}$ opening, filled with plating medium, and attached to a General Valve Picospritzer II via tubing. The driving force of the Picospritzer was $\mathrm{N}_{2}$ gas maintained at 40 pounds psi. Once a single growth cone was sighted, the pipette tip was positioned with a micromanipulator $25 \mu \mathrm{m}$ directly in front of the leading edge of the growth cone. The pipette was angled at $45^{\circ}$ to the dish surface and lowered into the dish medium until the tip was within $5 \mu \mathrm{m}$ of the dish surface. Medium was squirted through the pipette tip opening toward the growth cone at separate intervals beginning with a $10 \mathrm{msec}$ duration and increasing by $10 \mathrm{msec}$ with each trial. This "blasting" process was continued until the growth cone was completely dislodged from its original position on the dish surface, or until a blast duration of $200 \mathrm{msec}$ was reached. While the values obtained from these experiments cannot be related to any physical force such as microdynes (Heidemann et al., 1990), subjective impressions of the experimenters were that a blast of $40 \mathrm{msec}$ produced a relatively weak puff incapable of dislodging most cells or their processes and that a blast of $160 \mathrm{msec}$ produced a gale capable of detaching large adherent aggregates of cells from the dish. At least 20 growth cones were blasted from each of the five substrates using the same pipette tip. Dishes used for blasting were kept out of the incubator for a maximum of $20 \mathrm{~min}$. Only those experiments in which the same pipette was used for blasting of growth cones on all five sub- 

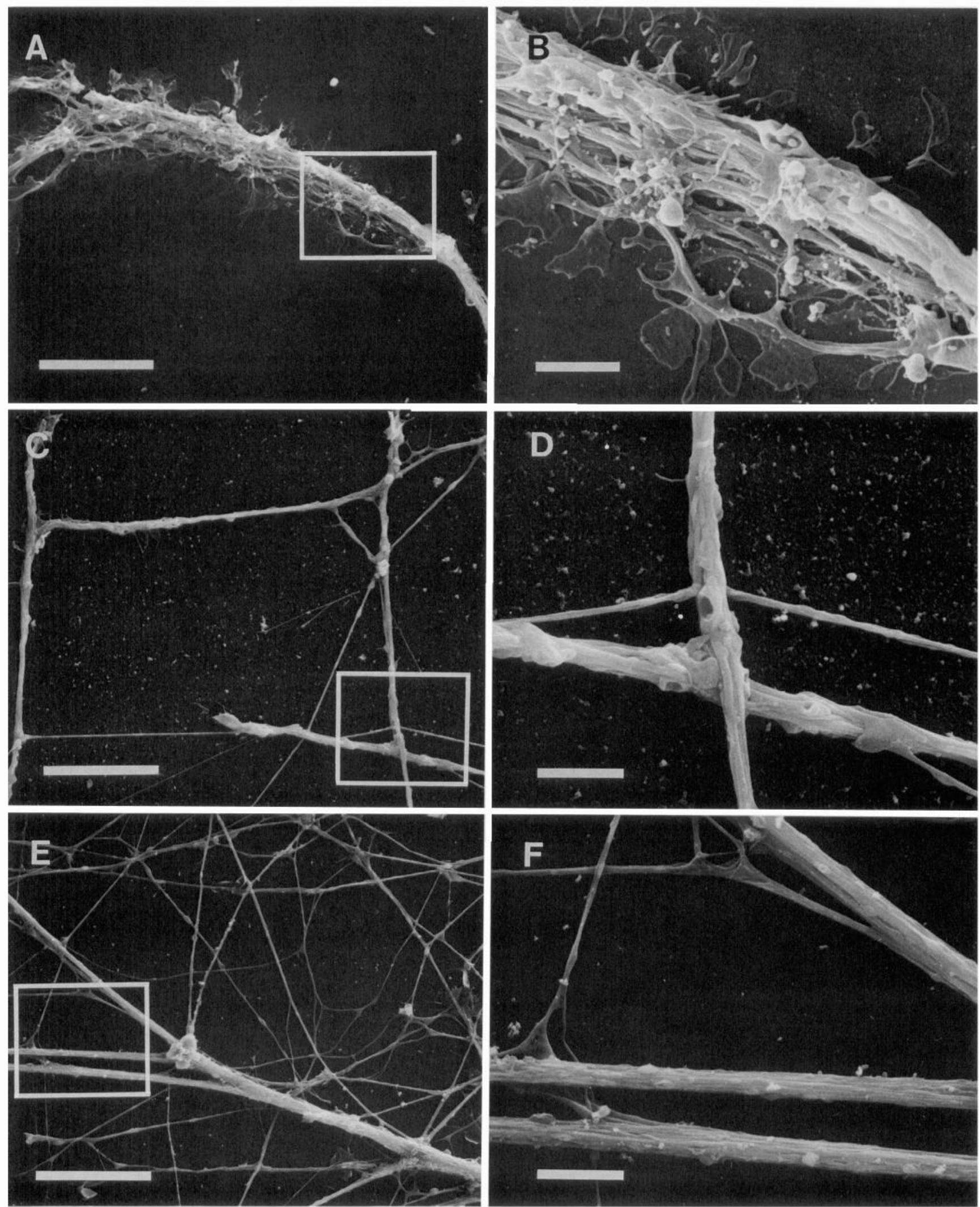

Figure 1. Scanning electron micrographs of patterns of fasciculation on different substrates: photographs on the right show higher magnifications of fields indicated in boxes in the photographs to the left. $A$ and $B$ show the dense mat of highly fasciculated neurites that grow on PL. $C$ and $D$ illustrate neurite fasciculation on laminin on NC. Bundles of fasciculated neurites are also evident on laminin on PL in $E$ and $F$. On N-cadherin $(G$ and $H)$, neurites are much more defasciculated than on laminin but can still form small bundles of tightly packed neurites (indicated with arrows in $H$ ). On $\mathrm{L} 1$ ( $I$ and $J$ ), even in regions of very dense neurite growth, the neurites rarely form compact bundles of neurites. Scale bars: $A, C, E, G$, and $I, 50 \mu \mathrm{m} ; B, D, F, H$, and $J, 10 \mu \mathrm{m}$. 

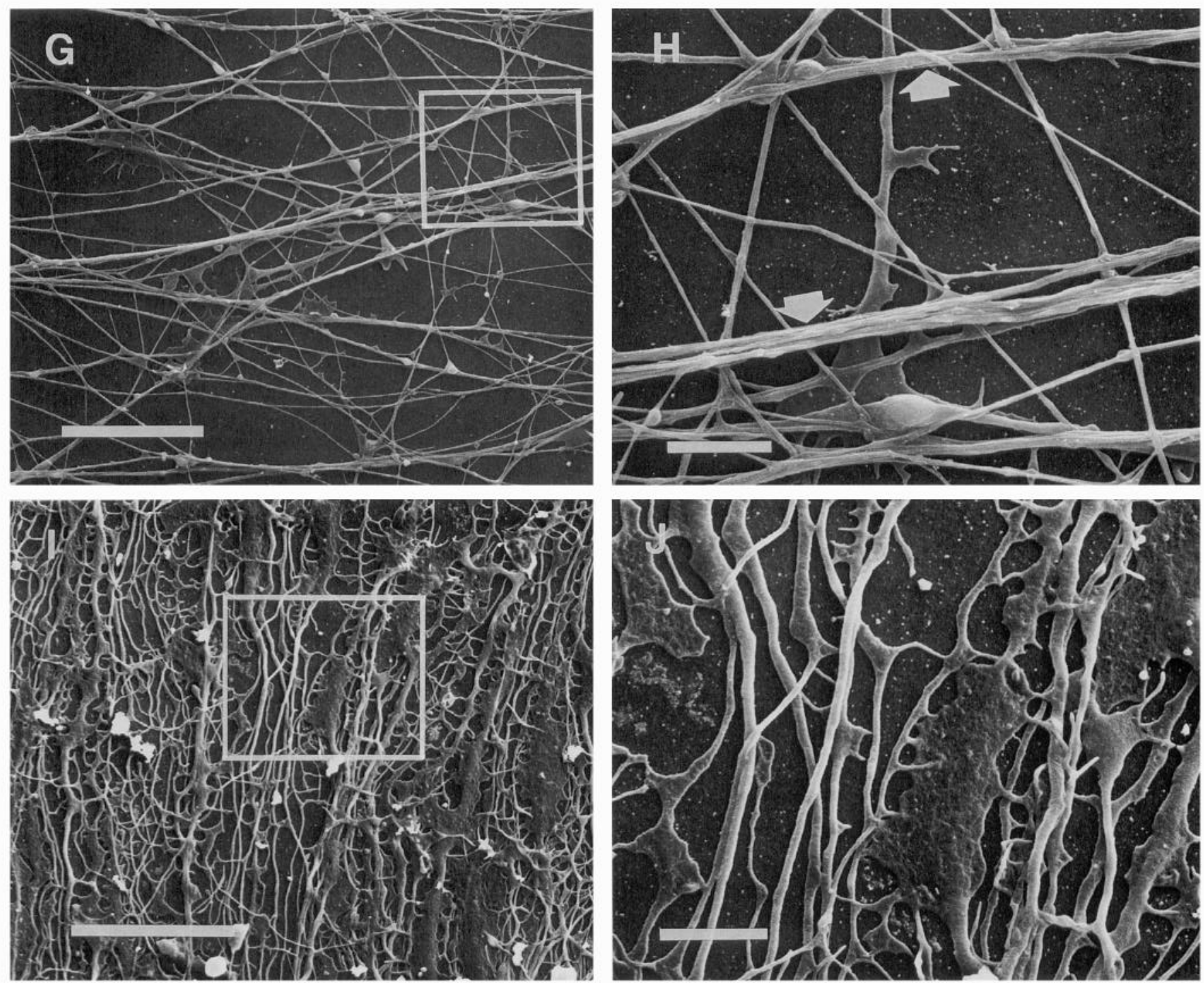

Figure 1. Continued.

strates were compiled for the final adhesivity assessment, although incomplete experiments (less than five substrates with the same pipette) also showed the same adhesivity trend. Pipette tips were examined after each blasting experiment, and the opening was measured.

Statistical analysis. The STATVIEW II statistical analysis program was used to analyze data from the growth rate and growth cone blasting experiments. Analysis of variance was done on both data sets. However, because the growth cone blasting experiments did not produce parametric data (McCall, 1970), pairwise comparisons were made between different substrates using the Mann-Whitney $U$ test.

Choice experiments. Alternating, parallel lanes of substrates were applied to tissue culture dishes with a silicone matrix generously donated by F. Bonhoeffer (Max-Planck-Institut für Entwicklungsbiologie, Tübingen, Germany), using a modification of a previously described procedure (Vielmetter et al., 1990). Briefly, the sterilized matrix was laid with the channel side facing the surface of an NC-coated Falcon $60 \mathrm{~mm}$ tissue culture dish. A $10 \mu$ laliquot of fluorescein-labeled substrate was injected into the inlet channel, incubated for $3 \mathrm{~min}$ at room temperature, and then replaced with a fresh substrate aliquot for five separate incubations of 15 total min. Fresh substrate exchanges were used to compensate for the small volume of the channels and to ensure even binding of the substrate to the dish. The substrate lanes were then saturated with inert protein by injecting a $20 \mu \mathrm{l}$ aliquot of sterile $4 \%$ bovine serum albumin (BSA) in $\mathrm{dH}_{2} \mathrm{O}$. Three separate injections of BSA at room temperature were used for a total incubation time of $10 \mathrm{~min}$. The lanes were then washed with three quick passes of $\mathrm{CMF}$, and the matrix was removed from the dish. The second substrate was applied to the sites between the channel lanes by spreading $20 \mu \mathrm{l}$ of substrate across the entire lane area. The second substrate was incubated in the covered dish for $15 \mathrm{~min}$ at room temperature, with frequent tilting of the dish to ensure even coating. Dishes were then rinsed with CMF and stored in plating medium in $37^{\circ} \mathrm{C}$ in $5 \% \mathrm{CO}_{2}, 95 \%$ air until used for plating (up to $16 \mathrm{hr}$ ). For each experiment, substrate 1 was applied to the channels in one dish with substrate 2 spread across the lane area, while substrate 2 was applied to the channels in a second dish with substrate 1 spread across the lane area. This provided a control that demonstrated that the order of substrate application did not affect neurite outgrowth preference. As with the conditions used in the other experiments described above, it was concluded that the substrate molecules were applied in high concentrations that would saturate the NC-coated plastic because 10 -fold dilutions of the substrates produced similar neurite growth characteristics. Inclusion of fluorescent markers with the different adhesion molecules showed that there was no detectable cross-contamination of adjacent lanes. Retinal explants were laid perpendicular to the substrate lanes in these dishes, and the neurite outgrowth was assessed at 24,48 , and $60 \mathrm{hr}$ after plating. At the completion of the experiment, the cultures were fixed with $1 \%$ paraformaldehyde, $0.01 \%$ glutaraldehyde in $0.1 \mathrm{M}$ phosphate buffer for $45 \mathrm{~min}$, followed by two $0.1 \mathrm{M}$ phosphate buffer rinses. The cultures were coverslipped, and the position of fluorescent substrate lanes was confirmed by viewing with an epifluorescence Leitz microscope. 
Table 1. Neurite growth rates on different substrates

\begin{tabular}{|c|c|c|c|c|c|}
\hline Rank & Substrate & $\begin{array}{l}\text { Maximum } \\
\text { growth rate } \\
\text { observed } \\
(\mu \mathrm{m} / \mathrm{hr})\end{array}$ & $\begin{array}{l}\text { Mean } \\
\text { growth rate } \\
(\mu \mathrm{m} / \mathrm{hr})\end{array}$ & $\begin{array}{l}\text { Standard } \\
\text { crror of } \\
\text { the mean } \\
\text { (SEM) }\end{array}$ & $\begin{array}{l}\text { Number of } \\
\text { growth } \\
\text { cones } \\
\text { examined } \\
\end{array}$ \\
\hline Slowest 1 & PL & 23 & 10 & 2 & 9 \\
\hline & L1 & 122 & 59 & 5 & 25 \\
\hline & N-Cadherin & 151 & 90 & 7 & 17 \\
\hline & Laminin-NC & 188 & 123 & 11 & 14 \\
\hline Fastest 5 & Laminin-PL & 230 & 136 & 15 & 13 \\
\hline
\end{tabular}

\section{Results}

Neurite growth rates. Growing neurites were observed with timelapse video equipment, and growth rates of the neurites were determined. Visual observation of explant cultures after 24 or $48 \mathrm{hr}$ in vitro revealed that neurites grew rapidly and for long distances on laminin and relatively poorly on PL, while the other substrates had intermediate growth promoting potentials. Measurements of growth rates confirmed these impressions of growth rates. The results shown in Table 1 indicate that neurite growth from retinal explants is slowest on PL, followed in order by $\mathrm{L} 1, \mathrm{~N}$-cadherin, laminin-NC, and laminin-PL. ANOVA showed that the growth rates on the five substrates are significantly different at the 0.05 level.

Fasciculation. Axons of projection neurons are usually found in bundles, growing in tracts in the CNS and in nerves in the PNS. The formation of these bundles is determined by choices the axons make; should they grow along other axons or should they grow through other terrains composed of extracellular matrix, glia, neuronal dendrites, or somas? When they choose to grow along other axons they form bundles or fascicles. The degree of fasciculation of neurites is extremely difficult to quantify both in vivo and in vitro. To assess fasciculation, we relied upon both qualitative and quantitative parameters: observation of growth cone encounters with neurite shafts during time-lapse video, examination of fixed cultures using both light microscopic and scanning electron microscopic techniques, and measuring fascicle width from scanning electron microscopic photographs. Time-lapse videos indicate that fasciculation is primarily determined by growth cone behavior. When a motile growth cone encounters a neurite, it may collapse and retract, it may adhere to the neurite and turn and grow along it forming a fascicle, or it may cross under or over the neurite and continue to grow along the substrate. We never observed neurite shafts that were adherent at one point to zip up from the point of contact to some distant point to form a fascicle.

Comparison of patterns of neurite fasciculation on different substrates (see Fig. 1) revealed distinctive growth patterns for the different substrates. On PL, the neurites emerged slowly from the explant as large, highly interwoven mats of neurites. Bundles containing many neurites would exit the mats and grow relatively short distances on the PL. The time-lapse videos revealed numerous lamellipodial and filopodial projections emerging from the growth cones and contacting either the PL or adjacent neurites. In the vast majority of the cases, the projections would fail to adhere to the PL but would usually form stable contacts with neuronal surfaces and therefore formed very compact fascicles. On laminin-NC, the neurites emerged rapidly from the explants and tended to grow as multiple bundles, with each bundle containing many neurites, although individual neurites were observed. The neurites on laminin-PL grew individually or as bundles with a few neurites. If a neurite growing on laminin encountered another neurite, it would frequently alter its course to grow along the precxisting ncuritc. $\mathrm{L} 1$ and $\mathrm{N}$-cadherin substrates produced the most defasciculated growth. Time-lapse videos revealed that on $\mathrm{L} 1$ the neurites would emerge as a broad front of independent growth cones growing in close proximity to each other but always choosing to grow along the substrate and not on other neurites. When examining fixed cultures with the light microscope, similar neurite growth patterns were observed on $\mathrm{N}$-cadherin and $\mathrm{Ll}$. The scanning electron microscope, however, revealed obvious differences between neurites on $\mathrm{L} 1$ and N-cadherin; $\mathrm{L} 1$ produced a significantly more defasciculated pattern of growth. On L1 the neurites flatten out (Payne et al., 1992), resulting in a slightly larger mean fascicle diameter on $\mathrm{L} 1$ compared to $\mathrm{N}$-cadherin. This is despite the fact that virtually all the neurites on $\mathrm{Ll}$ grew individually and not in fascicles while only about $20 \%$ of the neurites on N-cadherin were not in bundles with other neurites. The mean fasciclc diametcrs on the various substrates were (in $\mu \mathrm{m}$ ) L1, 0.884 $\pm 0.035 ; \mathrm{N}$-cadherin, $0.637 \pm 0.036$; laminin-PL, $1.384 \pm$ 0.103 ; laminin-NC, $1.705 \pm 0.121$ (mean $\pm \mathrm{SEM}$ ). $\mathrm{N}$-cadherin fascicles were significantly different from fascicles on laminin at the 0.0001 level.

Growth cone adhesion. Measurement of growth cone adhesion is difficult and may be influenced by factors such as growth cone size and shape, the number of receptors expressed on a growth cone for a given substrate, and the number of adherent filopodia emerging from the growth cone. While the adhesion data obtained in our experiments cannot be directly related to any physical force, the data can be used to rank relative adhesion. The results of five different experiments are presented in Table 2. L1 was most adhesive, followed by PL, laminin-PL, N-cadherin, and laminin-NC. The rankings obtained from the experiments were very reproducible. In the five experiments, there was only one instance, in experiment 3 , where there was a reversal in ranking between the two substrates, $\mathrm{N}$-cadherin and laminin-PL, that consistently had very similar adhesiveness. Pairwise comparisons of the substrates using the Mann-Whitney $U$ test revealed that different substrates had significantly different adhesiveness (at least at the 0.05 level) for all possible combinations except $\mathrm{N}$-cadherin versus laminin-PL. For this pair, only one of the five experiments met the 0.05 level criterion for being significantly different. Therefore, we conclude that $\mathrm{N}$ cadherin and laminin-PL have relatively similar adhesiveness for chick retinal ganglion cell growth cones. In separate exper- 


\section{A) THEORETICAL}

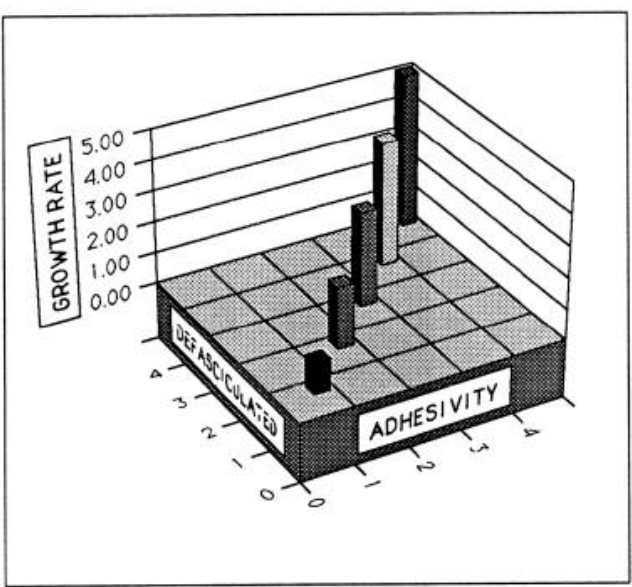

\section{B) OBSERVED}

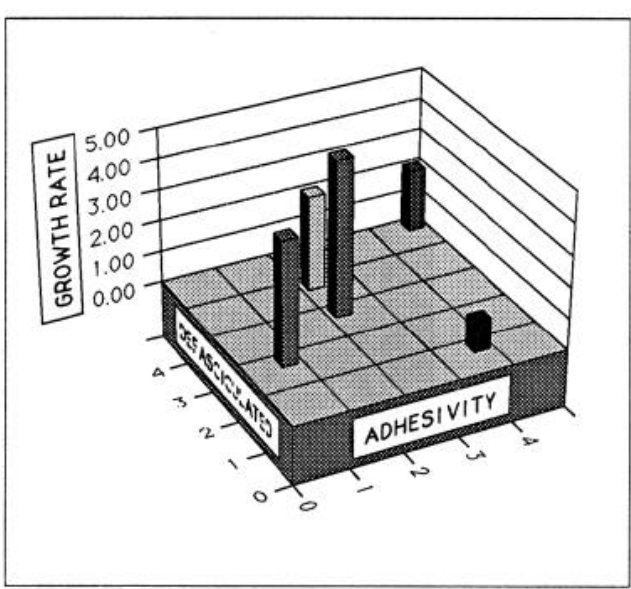

Figure 2. Correlation between substrate adhesiveness, neurite growth rates, and neurite fasciculation. $A$ shows the expected graph if increasing adhesiveness produced faster neurite growth and less fasciculation. $B$ shows the plot of the actual data obtained. See Results for discussion. iments, the adhesiveness values of rat $\mathrm{Ll}$ and chick L1 were not significantly different from each other (data not shown).

To determine if there is a correlation between growth cone adhesion to different substrates and either fasciculation or neurite growth rate, we chose to rank the results of the different experiments because the measures of adhesion and fasciculation do not meet the requirements of having magnitude, equal intervals, and absolute zero point that would permit direct correlation of the data (McCall, 1970). If substrate adhesiveness was positively correlated with neurite growth rate and defasciculation, then a three-dimensional plot of this would appear like that in Figure $2 A$. In our experiments, the least adhesive substrate (laminin-NC) was given a rank of 1 , the most adhesive substrate (L1) was given a rank of 5 , and the two substrates that were not significantly different from each other ( $\mathrm{N}$-cadherin and laminin-PL) were given equal ranks of 2.5 (Fig. $2 B$ ). For comparison, the mean growth rates on each substrate were used to rank the substrates. This analysis indicates there is a very poor correlation between growth cone adhesion and growth rates. Although there is a trend for the most adhesive substrates to have slower growth rates, neurites grew five times faster on the most adhesive substrate (L1) than on the fourth-ranked substrate (PL). To examine the relationship between adhesion and fasciculation, the substrate that produced the most defasciculated neurite growth (L1) was given a rank of 5 and the substrate that resulted in highly fasciculated growth (PL) was given a rank of 1 . Again, there was a very poor correlation between adhesivity and degree of fasciculation. Neurites do grow in a highly defas- ciculated manner on $\mathrm{L} 1$, the most adhesive substrate, yet neurites are the most fasciculated on PL, the second most adhesive substrate. Also, two substrates that were judged to be equally adhesive, $\mathrm{N}$-cadherin and laminin on PL, gave very different patterns of fasciculation.

Choice experiments. To determine the preferences of neurites for different substrates, we used a system that produces alternating lanes of different adhesion molecules (Vielmetter et al., 1990). The advantage of this system is that after application of the first adhesion molecule, it is possible to block the NC with a neutral protein before application of the second adhesion molecule to the alternate lanes. This greatly minimizes the amount of cross-contamination between the parallel lanes of substrates. Inclusion of fluorescently labeled proteins with the adhesion molecules indicated no cross-contamination of the substrates, as assessed with a fluorescence microscope. When neurites were given a choice between laminin and PL, L1 and PL, or N-cadherin and PL, the neurites always grew on the glycoproteins and not on the PL (data not shown). Therefore, neurites can show substrate preference in this assay. However, neurites showed relatively little selectivity when given choices between laminin and $\mathrm{N}$-cadherin, laminin and $\mathrm{L} 1$, or $\mathrm{Ll}$ and $\mathrm{N}$-cadherin, although there was a weak tendency for neurites to prefer $\mathrm{N}$-cadherin (Fig. 3). In all three cases, neurites were observed to grow from one substrate onto the second and then continue to an adjacent lane of the first substrate. The order of substrate application did not alter this result, consistent with a lack of crosscontamination of adjacent lanes of adhesion molecules. Due to

Table 2. Mean blast duration in milliseconds required to dislodge growth cones on different substrates

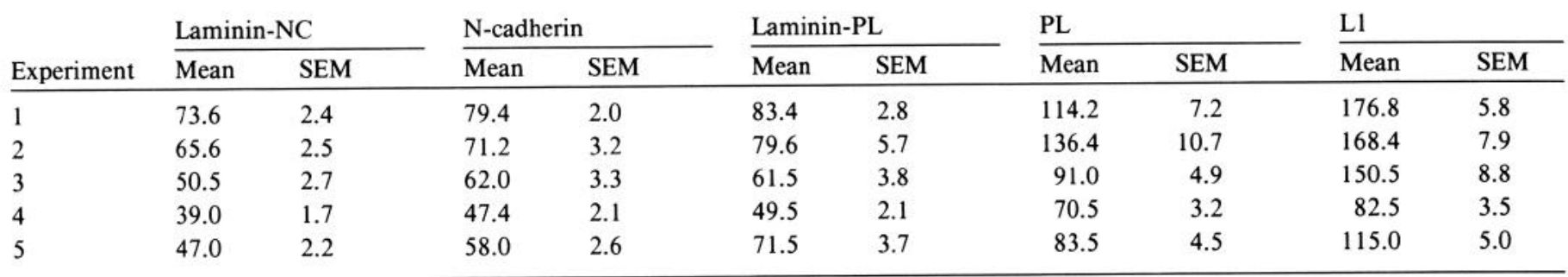




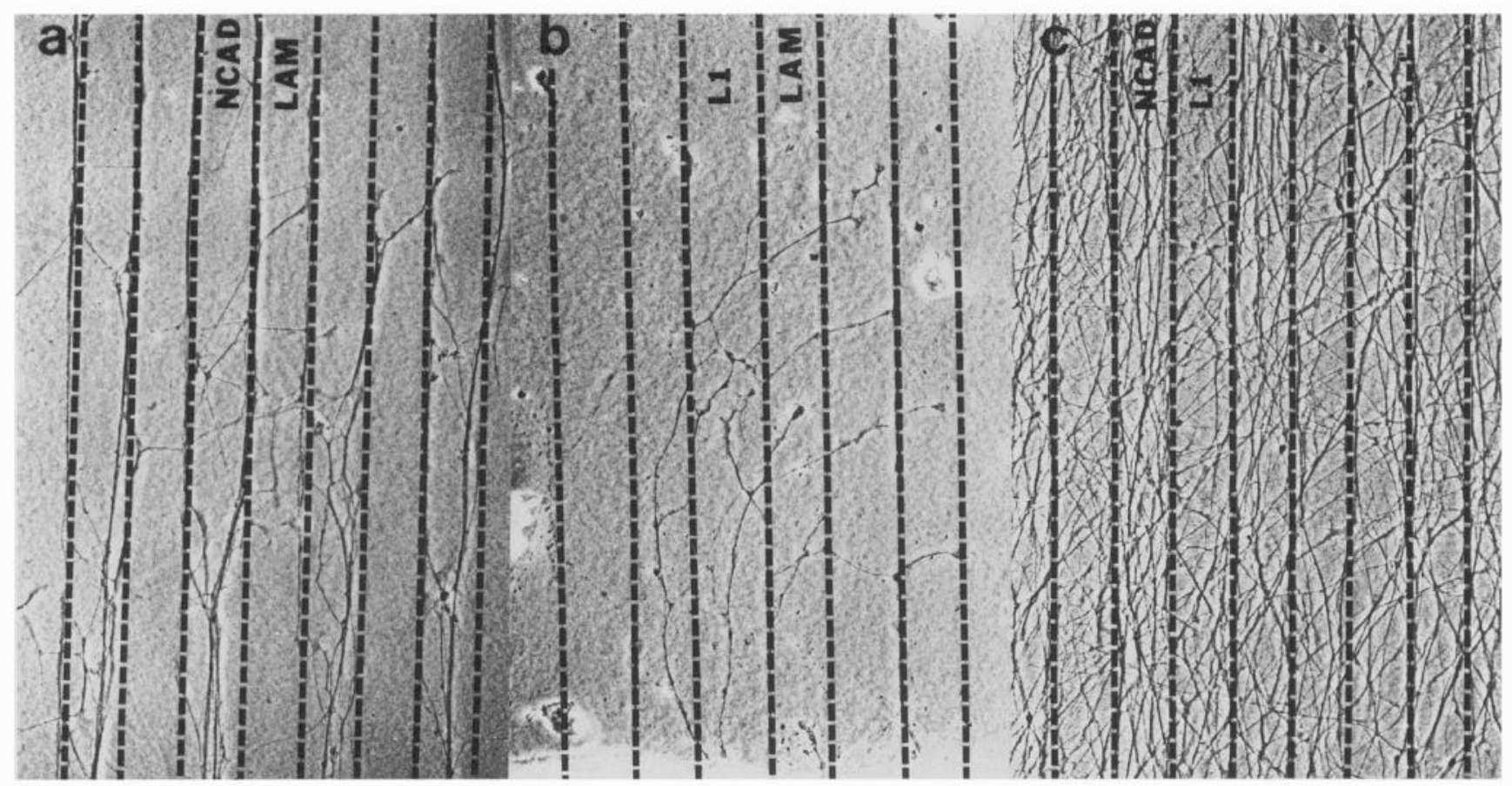

Figure 3. Parallel lane choice experiments. $a$, Neurites crossing alternating lanes of $\mathrm{N}$-cadherin and laminin. $b$, Neurites growing across alternating lanes of $\mathrm{L} 1$ and laminin. $c$, Neurites growing over alternating lanes of $\mathrm{N}$-cadherin and $\mathrm{L} 1$.

the much more adhesive nature of $\mathrm{L} 1$, the failure of neurites to localize on this substrate indicates that neurites are not selecting lanes by preference for more adhesive substrates.

\section{Discussion}

The mechanisms that determine growth cone choice of appropriate pathways through the developing CNS and embryo are undoubtedly complex and diverse (Walter et al., 1990; Reichardt and Tomaselli, 1991; Strittmatter and Fishman, 1991). There is general agreement that adhesion to a substrate is a prerequisite for neurite growth and that filopodial or lamellipodial attachment to a substrate may modulate the rate of neurite elongation (Lockerbie, 1987; Bray and Hollenbeck, 1988). A reasonable prediction from these observations is that neurites might grow more rapidly on more adhesive substrates. This is the case when artificial substrates such as tissue culture plastic, polyornithine and PL are compared (Letourneau, 1975). In our experiments, when naturally occurring substrates for neurite growth such as laminin, L1, and $\mathrm{N}$-cadherin were compared, there was, if anything, a negative correlation between substrate adhesivity and neurite growth rate. This is consistent with recent computer modeling of cell migration, which suggests that migration rates show a biphasic response to substrate adhesiveness. Migration rates increase as adhesiveness increases, but eventually the migration rates decrease to zero on very adhesive substrates (DiMilla et al., 1991). We observed, however, that the most adhesive substrate, $\mathrm{L}$, induced much higher neurite growth rates than the second most adhesive substrate, PL. One explanation for this observation is that cell surface receptors, such as L1 and integrins, may interact with cytoplasmic second messenger systems (Bixby, 1989; Schuch et al., 1989; Tomaselli and Reichardt, 1989) that actively regulate growth rate by controlling cytoskeletal assembly or disassembly or membrane in- sertion rates. PL would not be expected to interact with similar signal transduction systems.

Neurite fasciculation in vitro and in vivo has been described as the result of competition between axon-growth cone and axon-axon adhesion versus growth cone-substrate adhesion. For example, if growth cone-substrate adhesion is relatively high, then defasciculated neurites will be produced. Alternatively, if growth cone-axon adhesion is relatively high, then highly fasciculated neurites will appear (Rutishauser et al., 1988; Schubert and Klier, 1991). Data from experiments in which antibodies were employed to disrupt fasciculation have been interpreted to support this notion. For example, antibodies to NCAM and Ll cause defasciculation of neurites growing on laminin. Moreover, the ability of antibodies to disrupt fasciculation suggests that fasciculation depends on decisions made at the growth cone. The addition of antibodies during neurite growth inhibits fasciculation (Rathjen et al., 1987b) yet the same antibodies added at the same antibody concentration to established fascicles of live neurites, in the "cartwheel assay," do not result in a defasciculation of the neurite cables (Chang et al., 1987). The results of the present experiments indicate that substrate adhesivity is a poor predictor of the degree of neurite fasciculation. Two substrates with relatively similar adhesivity, $\mathrm{N}$-cadherin and laminin, produce very different patterns of fasciculation, with fascicles on laminin being on average twice as thick as those on $\mathrm{N}$-cadherin. Furthermore, growth cones growing on $\mathrm{L} 1$ and $\mathrm{N}$-cadherin both produce highly defasciculated patterns of neurite growth, yet $\mathrm{L} 1$ and $\mathrm{N}$-cadherin have extremely different adhesivities. Finally, PL, the second most adherent substrate, produces highly fasciculated outgrowth. Therefore, our findings suggest that the factors that cause neurite fasciculation are more complex than a simple competition between the adhesivity of growth cones for axons or the surrounding cells and matrix. 
The ability of neurites to discriminate between different patterned substrates in vitro is well documented. One of the earliest examples of discrimination was the demonstration that neurites prefer polyornithine or collagen to palladium (Letourneau, 1975). Based on such results, Letourneau (1975) proposed that neurites choose between two different pathways in vivo based on their relative adhesiveness. This notion has been very appealing, and investigators have attempted to infer the relative adhesiveness/ affinity of growth cones for particular substrates in vivo based on growth cone morphology and pathway choice (Nardi, 1983; Caudy and Bentley, 1986). Gunderson, however, has reported that dorsal root ganglion neurites prefer laminin to collagen yet growth cones bind more weakly to laminin (Gundersen, 1987) and have decreased areas of contact (Gundersen, 1988) when compared to collagen and fibronectin. In analogous experiments examining olfactory neuronal migration, Calof and Lander (1991) have shown that migrating neurons prefer laminin to fibronectin yet bind less tightly to laminin. These experiments indicate that when laminin and fibronectin are mixed, the laminin is antiadhesive, resulting in decreased adhesion even in the presence of excess fibronectin, probably by altering the cell's ability to bind to fibronectin and not by steric hindrance. There have been a number of experiments over the past few years (reviewed by Reichardt and Tomaselli, 1991) that indicate extracellular matrix components that support neurite growth are permissive but do not provide instructive information about direction of growth. Our results indicate this also may be true for $\mathrm{N}$-cadherin and L1. Our experiments show that CNS neurites can cross between L1, N-cadherin, and laminin, substrates with very different adhesivities for growth cones, going both from low adhesivity to high and vice versa. We also found that CNS neurites would not grow from $\mathrm{N}$-cadherin or $\mathrm{L} 1$ onto a substrate with an intermediate degree of adhesivity, PL. These results indicate that neurites, when confronted with naturally occurring substrates, do not select particular pathways exclusively by relative adhesivity.

Our results should not be interpreted as indicating that CAMs and extracellular matrix molecules are unimportant in guiding axons to their targets. Rather, some adhesion molecules such as laminin, $\mathrm{L} 1$, and $\mathrm{N}$-cadherin, which almost all axons can grow on, are likely to play an essential permissive role in providing pathways that promote rapid axon growth. Particular pathways might be selected by the expression of other receptors, such as TAG-1 (Dodd et al., 1988) or neurofascin (Rathjen et al., 1987a), on a subpopulation of axons that recognizes a given pathway. CAMs and substrate adhesion molecules (SAMs) also might act to signal particular pathways to certain classes of neurons by initiating active responses within their growth cones (Strittmatter and Fishman, 1991). Recently, integrins and immunoglobulin superfamily adhesion molecules have been implicated in activating conventional second messenger systems that regulate intracellular kinases (Bixby, 1989; Danilov and Juliano, 1989) and $\mathrm{Ca}^{2+}$ (Schuch et al., 1989). CAMs and SAMs are also thought to interact directly with the cytoskeleton (Horwitz et al., 1986; Pollerberg et al., 1986; Nagafuchi and Takeichi, 1988). In this way, growth cone adhesion to a substrate may be instructive and produce alterations in growth cone behavior in ways that direct growth along particular pathways. If this view is correct, then CAMs and SAMs should be viewed as cell surface receptors capable of signal transduction besides being mediators of cell adhesion.

\section{References}

Bixby JL (1989) Protein kinase C is involved in laminin stimulation of neurite outgrowth. Neuron 3:287-297.

Bixby JL, Zhang R (1990) Purified N-cadherin is a potent substrate for the rapid induction of neurite outgrowth. J Cell Biol 110:12531260.

Bray D, Hollenbeck PJ (1988) Growth cone motility and guidance. Annu Rev Cell Biol 4:43-61.

Buettner HM, Pittman RN (1991) Quantitative effects of laminin concentration on neurite outgrowth in vitro. Dev Biol 145:266-276.

Calof AL, Lander AD (1991) Relationship between neuronal migration and cell-substratum adhesion: laminin and merosin promote migration but are anti-adhesive. J Cell Biol 115:779-794.

Caudy M, Bentley D (1986) Pioneer growth cone morphologies proximal increases in substrate affinity within leg segments of grasshopper embryos. J Neurosci 6:364-379.

Chang S, Rathjen FG, Raper J (1987) Extension of neurites on axons is impaired by antibodies against specific neural cell surface glycoproteins. J Cell Biol 104:355-362.

Chiquet M, Nicholls JG (1987) Neurite outgrowth and synapse formation by identified leech neurones in culture. J Exp Biol 132:191206.

Danilov YN, Juliano RL (1989) Phorbol ester modulation of integrinmediated cell adhesion: a postreceptor event. J Cell Biol 108:19251933.

DiMilla PA, Barbee K, Lauffenburger DA (1991) Mathematical model for the effects of adhesion and mechanics on cell migration speed. Biophys J 60:15-37.

Dodd J, Morton SB, Karagogeos D, Yamamoto M, Jessel TM (1988) Spatial regulation of axonal glycoprotein expression on subsets of embryonic spinal neurons. Neuron 1:105-116.

Drazba J, Lemmon V (1990) The role of cell adhesion molecules in neurite outgrowth on Muller cells. Dev Biol 138:82-93.

Gundersen RW (1987) Response of sensory neurites and growth cones to patterned substrata of laminin and fibronectin in vitro. Dev Biol 121:423-431.

Gundersen RW (1988) Interference reflection microscopic study of dorsal root growth cones on different substrates: assessment of growth cone-substrate contacts. J Neurosci Res 21:298-306.

Gundersen RW, Barrett JN (1980) Characterization of the turning response of dorsal root neurites toward nerve growth factor. J Cell Biol 87:546-554.

Halfter W, Newgreen DF, Sauter J, Schwarz U (1983) Oriented axon outgrowth from avian embryonic retinae in culture. Dev Biol 95:5664.

Hatta K, Takeichi M (1986) Expression of N-cadherin molecules associated with early morphogenetic events in chick development. Nature 320:447-449.

Heidemann SR, Lamoureux P, Buxbaum RE (1990) Growth cone behavior and production of traction force. J Cell Biol 111:1949-1957.

Horwitz A, Duggan K, Buck C, Beckerle MC, Burridge K (1986) Interaction of plasma membrane fibronectin receptor with talin-a transmembrane linkage. Nature 320:531-533.

Lagenaur C, Lemmon V (1987) An L1-like molecule, the 8D9 antigen, is a potent substrate for neurite extension. Proc Natl Acad Sci USA 84:7753-7757.

Lein PJ, Higgins D (1989) Laminin and a basement membrane extract have different effects on axonal and dendritic outgrowth from embryonic rat sympathetic neurons in vitro. Dev Biol 136:330-345.

Lemmon V, McLoon S (1986) The appearance of an L1-like molecule in the chick primary visual pathway. J Neurosci 6:2987-2994.

Lemmon V, Farr K, Lagenaur C (1989) L1 mediated axon outgrowth occurs via a homophilic binding mechanism. Neuron 2:1597-1603.

Letourneau PC (1975) Cell-to-substratum adhesion and guidance of axonal elongation. Dev Biol 44:92-101.

Lockerbie RO (1987) The neuronal growth cone: a review of its locomotory, navigational and target recognition capabilities. Neuroscience 20:719-729.

McCall RB (1970) Fundamental statistics for psychology. New York: Harcourt, Brace \& World.

McKenna MP, Raper JA (1988) Growth cone behavior on gradients of substratum bound laminin. Dev Biol 130:232-236.

Nagafuchi A, Takeichi M (1988) Cell binding function of E-cadherin is regulated by the cytoplasmic domain. EMBO J 7:3679-3684. 
Nardi JB (1983) Neuronal pathfinding in developing wings of the moth Manduca sexta. Dev Biol 95:163-174.

Payne HR, Burden S, Lemmon V (1992) Modulation of growth cone morphology by substrate-bound adhesion molecules. Cell Motil Cytoskel 21:65-73.

Pollerberg G, Schachner M, Davoust J (1986) Differentiation statedependent surface mobilities of two forms of neural cell adhesion molecule. Nature 324:462-465.

Postek MT, Howard KS, Johnson AH, McMichael KL (1980) Scanning electron microscopy: a student's handbook. Burlington, VT: Ladd Research Industries.

Rathjen FG, Wolff JM, Chang S, Bonhoeffer F, Raper J (1987a) Neurofascin: a novel chick cell-surface glycoprotein involved in neuriteneurite interactions. Cell 51:841-849.

Rathjen FG, Wolff JM, Frank R, Bonhoeffer F, Rutishauser U (1987b) Membrane glycoproteins involved in neurite fasciculation. J Cell Biol 104:343-353.

Reichardt LF, Tomaselli KJ (1991) Extracellular matrix molecules and their receptors-functions in neural development. Annu Rev Neurosci 14:531-570.

Ross WN, Arechiga H, Nicholls JG (1988) Influence of substrate on the distribution of calcium channels in identified leech neurons in culture. Proc Natl Acad Sci USA 85:4075-4078.

Rutishauser U, Acheson A, Hall AK, Mann DM, Sunshine J (1988) The neural cell adhesion molecule (NCAM) as a regulator of cell-cell interactions. Science 240:53-57.

Sanes JR (1989) Extracellular matrix molecules that influence neural development. Annu Rev Neurosci 12:491-516.
Schubert D, Klier FG (1991) Substratum regulation of neurite fasciculation. Brain Res 549:305-310.

Schuch U, Lohse MJ, Schachner M (1989) Neural cell adhesion molecules influence second messenger systems. Neuron 3:13-20.

Schwab ME, Schnell L (1991) Channeling of developing rat corticospinal tract axons by myelin-associated neurite growth inhibitors. J Neurosci 11:709-721.

Snow DM, Lemmon V, Carrino DA, Caplan AI, Silver J (1990) Molecular basis of neurite outgrowth inhibition by glia: the role of keratin sulfate/chondrotin sulfate proteoglycan in vitro. Exp Neurol 109:111130.

Strittmatter SM, Fishman MC (1991) The neuronal growth cone as a specialized transduction system. Bioessays 13:127-134.

Tomaselli KJ, Reichardt LF (1989) Integrins, cadherins, and cell adhesion molecules of the immunoglobulin superfamily: neuronal receptors that regulate axon growth and guidance. In: The assembly of the nervous system (Landmesser L, ed), pp 81-108. New York: Liss.

Vielmetter J, Stolze B, Bonhoeffer F, Stuermer CAO (1990) In vitro assay to test differential substrate affinities of growing axons and migratory cells. Exp Brain Res 81:283-287.

Walter J, Kern-Veits B, Huf J, Stolze B, Bonhoeffer F (1987) Recognition of position-specific properties of tectal cell membranes by retinal axons in vitro. Development 101:685-696.

Walter J, Allsopp TE, Bonhoeffer F (1990) A common denominator of growth cone guidance and collapse. Trends Neurosci 13:447-452. 